\title{
Nudging consumers towards healthier choices: a systematic review of positional influences on food choice
}

\author{
Tamara Bucher ${ }^{1,2 *}$, Clare Collins ${ }^{1,2}$, Megan E. Rollo ${ }^{1,2}$, Tracy A. McCaffrey ${ }^{3}$, Nienke De Vlieger ${ }^{1,2}$, \\ Daphne Van der Bend ${ }^{1,2}$, Helen Truby ${ }^{3}$ and Federico J. A. Perez-Cueto ${ }^{4}$ \\ ${ }^{1}$ Nutrition and Dietetics, School of Health Sciences, Faculty of Health and Medicine, The University of Newcastle, University \\ Drive, Newcastle, Callaghan NSW 2300, Australia \\ ${ }^{2}$ Priority Research Centre in Physical Activity and Nutrition, The University of Newcastle, University Drive, Newcastle, \\ Callaghan NSW 2300, Australia \\ ${ }^{3}$ Department of Nutrition and Dietetics, School of Clinical Sciences, Monash University, Melbourne, VIC 3168, Australia \\ ${ }^{4}$ Sensory and Consumer Section, Department of Food Science, University of Copenhagen, Rolighedsvej 26, \\ 1958 Frederiksberg C, Denmark.
}

(Submitted 26 August 2015 - Final revision received 21 March 2016 - Accepted 22 March 2016)

\begin{abstract}
Nudging or 'choice architecture' refers to strategic changes in the environment that are anticipated to alter people's behaviour in a predictable way, without forbidding any options or significantly changing their economic incentives. Nudging strategies may be used to promote healthy eating behaviour. However, to date, the scientific evidence has not been systematically reviewed to enable practitioners and policymakers to implement, or argue for the implementation of, specific measures to support nudging strategies. This systematic review investigated the effect of positional changes of food placement on food choice. In total, seven scientific databases were searched using relevant keywords to identify interventions that manipulated food position (proximity or order) to generate a change in food selection, sales or consumption, among normal-weight or overweight individuals across any age group. From 2576 identified articles, fifteen articles comprising eighteen studies met our inclusion criteria. This review has identified that manipulation of food product order or proximity can influence food choice. Such approaches offer promise in terms of impacting on consumer behaviour. However, there is a need for high-quality studies that quantify the magnitude of positional effects on food choice in conjunction with measuring the impact on food intake, particularly in the longer term. Future studies should use outcome measures such as change in grams of food consumed or energy intake to quantify the impact on dietary intake and potential impacts on nutrition-related health. Research is also needed to evaluate potential compensatory behaviours secondary to such interventions.
\end{abstract}

Key words: Nudging: Choice architecture: Food position: Proximity: Order: Food choices: Environmental influences

In recent years, there has been a shift away from solely targeting individuals to change their eating behaviours to an approach that addresses wider, population-level factors and involves other environmental components and stakeholders ${ }^{(1)}$. Foodscapes $^{(2)}$ and food environments contribute to the so-called 'obesogenic environment' ${ }^{,(1,3)}$ and influence food choices. Epidemiological data suggest that numerous small changes towards a healthier behaviour such as improving diet quality have the potential to have a positive impact on reducing mortality risk ${ }^{(4)}$. Most healthy eating interventions in Europe have been successful in providing consumers with information to enable them to make better-informed food choices ${ }^{(5)}$. Although they have been successful in creating awareness among consumers, there has only been modest success in terms of actual lifestyle changes and measurable health indicators in the sample populations, such as weight reduction ${ }^{(6)}$. Individualised behaviour change is ineffective unless it becomes habit forming, which requires support and reinforcement through structural or environmental change so that the new behaviour is sustained. Although behavioural economics have impacted on some policy interventions, the case for foodrelated interventions remains under development, constituting a promising area that could potentially achieve high social benefits $^{(7,8)}$

Therefore, innovative intervention strategies that are able to effectively improve food behaviours, dietary intake and impact on health status need to be investigated and implemented. The majority of interventions have an underlying assumption that people make conscious and reasoned food choices, most of the time ${ }^{(9)}$. This paradigm has been questioned following the limited impact of information-based campaigns in achieving behaviour change, and the subsequent rise in the prevalence of obesity and other chronic diseases ${ }^{(10)}$. Furthermore, current paradigms place the burden and responsibility for all food 
choices on the individual, with the justification that everyone is free to make healthy choices once informed ${ }^{(6,11)}$.

Dietary habits and food choices are the result of decisions and actions that are based on routines that require very little active decision making as well as reflective, elaborate decision making where choice options are carefully considered. Choice architecture, inspired by behavioural economics, describes the way in which decisions are influenced based on how choices are presented within meal environments ${ }^{(12)}$. The meal environment has been defined as the room, the people, the food, the atmosphere and the management system, particularly when eating out of home. This suggests that the meal environment can be modified to be more or less conducive to support the required behaviour and as such may lead to weight changes, either through promotion of healthier choices or through decreased intake ${ }^{(12-15)}$.

Choice architecture is often used interchangeably with other terms such as nudging, libertarian paternalism and behavioural economics. Choice architecture is a subset of non-regulatory behavioural interventions. Choice architecture can include one or more of the following: provision of information (e.g. to activate a rational choice), changes in the physical environment (e.g. light, décor, placement, etc.), changes in the default policy (e.g. pre-weighed salad portions $v$. free serving of a salad bowl) and use of social norms and salience (e.g. comparison with average consumers ${ }^{(16)}$. Nudging has been defined as any aspect of the choice architecture that alters people's behaviour in a predictable way without forbidding any options or significantly changing their economic incentives ${ }^{(15)}$. Within the public health nutrition area, this could mean altering the food environment, such as product placement or labelling or even encouraging consumers to sit together for their meal (social facilitation). Furthermore, nudging interventions consist of provision of information, changes to physical environment, changes to the default policy and the use of social norms and salience ${ }^{(16)}$.

Previous studies have shown that nudging practices are promising measures that can be used to support the promotion of healthy eating. An example of nudging is that by changing the size of dishware, portion sizes may be reduced leading to unconscious changes in actual food intake ${ }^{(17)}$ and meal composition $^{(18)}$. Similarly, food positioning is thought to influence food choice. Studies have shown that people eat more unhealthy food such as chocolate if it is located more prominently ${ }^{(19)}$. However, it is less clear whether minor changes in food position or item placement, which are not accompanied by changes in effort, also promote healthier food choices ${ }^{(13,20)}$.

Existing systematic reviews that have investigated the effectiveness of choice architecture interventions have mainly focused on the effectiveness of labelling and prompting ${ }^{(21,22)}$. However, these types of interventions are more closely related to the traditional behavioural interventions of information giving $^{(23)}$. To date, there is no systematic review that has assessed the influence of food placement within microenvironments on product choice and on food intake ${ }^{(23)}$. This information is relevant for the support of public health interventions and relevant for operations in the food service sector.

The aims of this systematic review were to evaluate published articles that have investigated the effect of positional changes within microenvironments on food choice by healthy weight and overweight individuals across all age groups and to derive recommendations for future research in the area.

For the purpose of this review, we have defined a nudging intervention as any intervention that involves altering the non-economic properties or placement of objects or stimuli within micro-environments with the intention of changing health-related behaviour. Such interventions are implemented within the same micro-environment in which the target behaviour is performed and require minimal conscious engagement. In principle, these interventions can influence the behaviour of many people simultaneously, and they are not targeted or tailored to specific individuals (adapted from Hollands et al. ${ }^{(23)}$ ). The present review focuses on positional changes that affect immediate food consumption or choice decisions of individuals (e.g. eating out of home in a food service outlet), rather than the consumption pattern of a family or a household over time, as it would be the case in 'assortment structure' experiments within supermarket settings.

\section{Methods}

Details of the protocol for this systematic review were registered on the International Prospective Register of Systematic Reviews (PROSPERO) and can be accessed at http://www.crd.york.ac. uk/PROSPERO/display_record.asp?ID=CRD42015016277

\section{Criteria for study inclusion}

The PICOS (Problem, Intervention, Comparison, Outcome, Setting) approach ${ }^{(24)}$ was used to frame the research question. We defined 'food choice' as all outcome measures that assessed food selection or probability of food choice, including product sales and food consumption (in grams or energy intake). Positional changes were defined as all manipulations of food order or variations in the distance of food placement relative to consumers within microenvironments. Microenvironments were defined as the immediate surroundings of the individuals, such as within the home, workplace or cafeterias ${ }^{(25)}$.

The types of studies to be included were randomisedcontrolled trials/experiments, pre-post experimental studies, quasi experiments and naturalistic observations where at least one research aim was to assess the influence of food positioning within a microenvironment on food choice (selection) or sales (grams, number) and intake (grams, energy).

Studies where multiple variables were manipulated simultaneously along with the food position were not included. For example, studies where foods were added or removed from the selection or where portion sizes of healthy or unhealthy offers were altered along with a positional change were excluded. Study participants included only healthy, normal-weight or overweight/obese individuals. There was no age restriction with studies on both children and adults included. The search included full-text articles that were published in peer-reviewed journals in the English language.

\section{Literature search}

A systematic search was conducted using electronic databases (Medline, Pre-Medline, Embase, CINAHL, Scopus, The Cochrane 
Library and PsycINFO) until February 2015. No limit was placed on publication date. The search term list included the following items: choice architecture OR accessib* OR nudg* OR position* OR (serving AND (direction OR distance)) OR proximity OR distance AND food OR diet OR food choice OR energy intake OR caloric restriction OR fruits OR vegetables OR health* OR food choice. Reference lists of included articles and key reviews in the area were also manually searched for additional articles.

\section{Review procedure}

Two independent reviewers (T. B. and N. D. V./D. V. d. B.) screened the titles and abstracts of all search results. Full texts of all papers that appeared to potentially meet the inclusion criteria were retrieved. The retrieved full texts were assessed by two independent reviewers (T. B. and N. D. V.) to determine inclusion. In case of disagreement, a third independent reviewer made the final decision (M. E. R.).

\section{Data extraction and synthesis}

Quantitative data on study participants (age, sex, weight status), the design (type of study, setting, manipulated variables) and the outcomes (finding, main effect, conclusions) from the included articles were extracted by T. B. and checked by M. E. R. To distinguish between the magnitude of the change in effort that was involved in the intervention, we differentiated between minor changes (mere order change or very small distance change within reach), medium (change of position to food that required only a small effort, e.g. standing up, bending down) or major positional changes (manipulations that involved a major increase/reduction in effort, e.g. walking across a room).

\section{Quality assessment of included studies}

The quality of the included studies was assessed by two independent reviewers (T. A. M. and H. T.) using the review evidence analysis manual published by the Academy of Nutrition and Dietetics ${ }^{(26)}$. The quality scores can be found in the online Supplementary Table S1.

\section{Results}

The database search identified 2540 unique entries, which were combined with another thirty-six articles of interest that were identified by screening reference lists. A total of sixty-two fulltext articles were retrieved and assessed against the inclusion criteria. In total, fifteen articles, comprising eighteen studies, met the inclusion criteria and the data from these were extracted and evaluated in this review (see Fig. 1).

The majority ( $n$ 10) of studies were conducted in the USA; seven were conducted in Europe, of which four were conducted in the Netherlands. In one study, the country was not reported $^{(27)}$. There was only one study on children ${ }^{(28)}$. Moreover, ten studies were conducted with university students or staff, and for five studies the subjects were customers of hospital cafeterias. Only one study was conducted in an Army

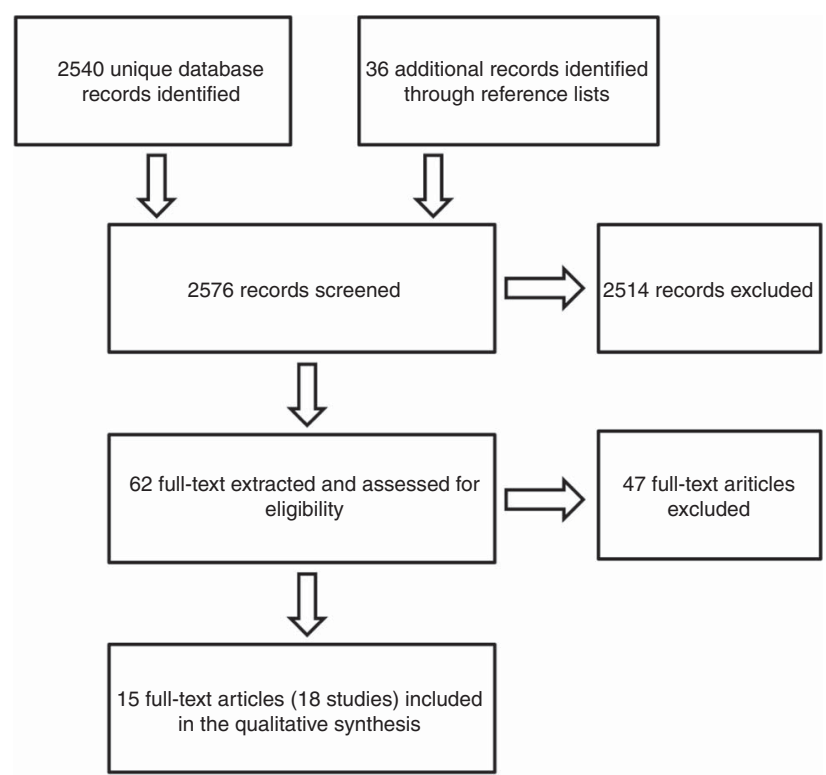

Fig. 1. Flow of information through the different phases of the review.

research centre ${ }^{(29)}$ and one was conducted with attendees of a health conference ${ }^{(30)}$.

The foods involved in the studies varied and included single healthy or unhealthy items (water, fruit and vegetable, cereal bars, chocolate candy or crackers) to more complex selections within canteen buffets with between eight and eleven products repositioned.

Among all, seven studies reported participants' weight status; however, only two considered it in the analysis ${ }^{(27,31)}$. Levitz ${ }^{(27)}$ reported that a change in dessert order affected normal-weight and overweight people differently. In particular, the author found that obese adults selected a greater amount of lowenergy dessert if it was made more salient. No changes were observed if the high-energy desserts were made more salient $^{(27)}$.

The characteristics of the included studies are summarised in Table 1.

Of the eighteen studies that were included, only one received a positive quality rating ${ }^{(32)}$, with fourteen studies being assessed as neutral and three as negative, because study procedures were not described in detail and several validity questions could not be answered clearly (online Supplementary Table S1).

Of the eighteen studies, nine investigated the effect of distance/proximity changes on food choice, such as placing unhealthy foods further from the consumer. The other half assessed whether changes in product order, such as, for example, the food sequence on a buffet, could have a beneficial influence on food selection.

In summary, sixteen of the eighteen studies concluded that positional changes had a positive influence on food choice. The only two studies that did not find an effect manipulated the product order of snacks on a computer screen (Van Kleef Study 1), as well as within a shelf at a checkout counter in a cafeteria (Van Kleef Study 2). However, in the field study, they found a trend towards sales of healthy food being positively affected ${ }^{(32)}$. 


\section{N British Journal of Nutrition}

Table 1. Characteristics of included studies ( $n$ 18) assessing the effect of positional changes in the microenvironment on food choice

\begin{tabular}{|c|c|c|c|c|c|c|c|}
\hline Author, year & Type of study & Type of nudge & Setting & Country & Subjects & Subject age (years) & Subject weight status \\
\hline Engell et al., 1996 & $\begin{array}{l}\text { Experimental between- } \\
\text { subjects design }\end{array}$ & $\begin{array}{l}\text { Distance/ } \\
\text { proximity }\end{array}$ & $\begin{array}{l}\text { Field study: Army } \\
\text { research centre dining } \\
\text { hall }\end{array}$ & USA (Boston) & $\begin{array}{l}\text { Employees of US Army } \\
\text { Natick Research Centre, } \\
n 36\end{array}$ & $39 \cdot 5 \pm 13 \cdot 2$ & $\begin{array}{l}\text { Normal weight } \\
\quad(181 \cdot 6 \pm 30 \cdot 7) \text { pounds, } \\
70 \cdot 6 \pm 2 \cdot 4 \text { inches })\end{array}$ \\
\hline $\begin{array}{l}\text { Maas et al., } 2012 \\
\text { (study 1) }\end{array}$ & $\begin{array}{l}\text { Experimental between- } \\
\text { subjects design }\end{array}$ & $\begin{array}{l}\text { Distance/ } \\
\text { proximity }\end{array}$ & Laboratory & $\begin{array}{l}\text { The Netherlands } \\
\text { (Utrecht) }\end{array}$ & $\begin{array}{l}77 \text { females recruited on } \\
\text { campus }\end{array}$ & $17-38$ & $\begin{array}{l}\text { Normal weight (BMl: } \\
\left.22.4 \pm 2.96 \mathrm{~kg} / \mathrm{m}^{2}\right)\end{array}$ \\
\hline $\begin{array}{l}\text { Maas et al., } 2012 \\
\quad \text { (study 2) }\end{array}$ & $\begin{array}{l}\text { Experimental between- } \\
\text { subjects design }\end{array}$ & $\begin{array}{l}\text { Distance/ } \\
\text { proximity }\end{array}$ & Laboratory & $\begin{array}{l}\text { The Netherlands } \\
\text { (Utrecht) }\end{array}$ & $\begin{array}{l}54 \text { females recruited on } \\
\text { campus }\end{array}$ & $17-38$ & $\begin{array}{l}\text { Normal weight (BMI: } \\
\left.20.89 \pm 2 \cdot 16 \mathrm{~kg} / \mathrm{m}^{2}\right)\end{array}$ \\
\hline $\begin{array}{l}\text { Meiselman et al., } \\
1994 \text { (study 1) }\end{array}$ & $\begin{array}{l}\text { Experiment (repeated } \\
\text { measures possible) }\end{array}$ & $\begin{array}{l}\text { Distance/ } \\
\text { proximity }\end{array}$ & $\begin{array}{l}\text { Field study: university } \\
\text { cafeteria }\end{array}$ & $\begin{array}{l}\text { England } \\
\text { (Bournemouth) }\end{array}$ & $\begin{array}{l}\text { Customers in university } \\
\text { cafeteria; } 43 \text { students } \\
334 \text { meals }\end{array}$ & $\begin{array}{l}18 \text { years, } 4 ; 19 \text { years, } \\
15 ; 20 \text { years, } 12 ; \\
21 \text { years, } 7 \text {; over } \\
21.5 \text { years }\end{array}$ & N/A \\
\hline $\begin{array}{l}\text { Meiselmann et al., } \\
1994 \text { (study 2) }\end{array}$ & $\begin{array}{l}\text { Experiment (repeated } \\
\text { measures possible) }\end{array}$ & $\begin{array}{l}\text { Distance/ } \\
\text { proximity }\end{array}$ & $\begin{array}{l}\text { Field study: university } \\
\text { cafeteria }\end{array}$ & $\begin{array}{l}\text { England } \\
\text { (Bournemouth) }\end{array}$ & $\begin{array}{l}\text { Meals of customers in } \\
\text { university cafeteria; } 60 \\
\text { students ( } 36 \text { male) who } \\
\text { consumed potato chips at } \\
\text { baseline }\end{array}$ & $\begin{array}{l}\text { Between } 18 \text { and } \\
62 \text { years }\end{array}$ & N/A \\
\hline $\begin{array}{l}\text { Musher-Eizenman } \\
\text { et al., } 2010\end{array}$ & Correlation analysis & $\begin{array}{l}\text { Distance/ } \\
\text { proximity }\end{array}$ & $\begin{array}{l}\text { Field study: child day } \\
\text { care }\end{array}$ & USA (Ohio) & 46 children & $\begin{array}{l}6 \cdot 3 \pm 2 \cdot 3 \text {, range: } \\
3 \cdot 4-11\end{array}$ & $\begin{array}{l}\text { 8th to } 98 \text { percentile } \\
\text { (M=65th), } 25 \% \\
\text { overweight (85th } \\
\text { percentile and higher) }\end{array}$ \\
\hline Privitera et al., 2010 & $\begin{array}{l}\text { Between-subjects } \\
\text { experiment }\end{array}$ & $\begin{array}{l}\text { Distance/ } \\
\text { proximity }\end{array}$ & Laboratory & $\begin{array}{l}\text { USA (St. } \\
\text { Bonaventure, } \\
\text { NY) }\end{array}$ & $\begin{array}{l}96 \text { (24 male) university } \\
\text { students }\end{array}$ & $\begin{array}{l}\text { Variation } 1 \text { : } \\
\text { 19.9 } 1 \cdot 1 \text {; variation } \\
2: 20 \cdot 1 \pm 16\end{array}$ & $\begin{array}{l}\text { BMl: } 26 \cdot 9 \pm 3 \cdot 8 \text { and } \\
26 \cdot 4 \pm 4 \mathrm{~kg} / \mathrm{m}^{2} \text { (mean } \\
\text { overweight) }\end{array}$ \\
\hline Privitera et al., 2014 & $\begin{array}{l}\text { Between-subjects } \\
\text { experiment }\end{array}$ & $\begin{array}{l}\text { Distance/ } \\
\text { proximity }\end{array}$ & Laboratory & $\begin{array}{l}\text { USA (St. } \\
\text { Bonaventure, } \\
\text { NY) }\end{array}$ & $\begin{array}{l}56 \text { university students } \\
(26 \text { male })\end{array}$ & $19 \pm 0.9$ & $\begin{array}{l}\text { BMI: } 26 \cdot 0 \pm 3 \cdot 8 \mathrm{~kg} / \mathrm{m}^{2} \\
21 \text { overweight, } \\
15 \text { obese }\end{array}$ \\
\hline $\begin{array}{l}\text { Wansink et al., } \\
2006\end{array}$ & Within-subjects experiment & $\begin{array}{l}\text { Distance/ } \\
\text { proximity }\end{array}$ & $\begin{array}{l}\text { Field study: offices at } \\
\text { university }\end{array}$ & USA (Illinois) & $\begin{array}{l}40 \text { female university staff } \\
\text { members }\end{array}$ & $42 \cdot 2 \pm 11 \cdot 3$ & $N / A$ \\
\hline $\begin{array}{l}\text { Keller \& Bucher, } \\
2014\end{array}$ & $\begin{array}{l}\text { Experimental between- } \\
\text { subjects design }\end{array}$ & $\begin{array}{l}\text { Order/ } \\
\text { accessibility }\end{array}$ & $\begin{array}{l}\text { Field study: university } \\
\text { campus }\end{array}$ & $\begin{array}{l}\text { Switzerland } \\
\text { (Zurich) }\end{array}$ & $\begin{array}{l}120 \text { students ( } 60 \text { male, age } \\
24 \pm 3 \text { years) }\end{array}$ & $24 \pm 3$ & N/A \\
\hline Levitz, 1976 & $\begin{array}{l}\text { Naturalistic observation, } \\
\text { experiment (repeated } \\
\text { measures possible) }\end{array}$ & $\begin{array}{l}\text { Order/ } \\
\text { accessibility }\end{array}$ & $\begin{array}{l}\text { Field study: hospital } \\
\text { cafeteria }\end{array}$ & $\mathrm{N} / \mathrm{A}$ & $\begin{array}{l}\text { Customers in hospital } \\
\text { cafeteria. } 3267 \\
\text { observations. Only } \\
\text { choices of normal-weight } \\
(n \text { 2385) and obese } \\
(n \text { 425) subjects were } \\
\text { analysed }\end{array}$ & $\mathrm{N} / \mathrm{A}$ & $\begin{array}{l}\text { Normal weight and } \\
\text { overweight } \\
\text { (classification by } \\
\text { trained observers) }\end{array}$ \\
\hline Levy et al., 2012 & $\begin{array}{l}\text { Longitudinal study pre-post } \\
\text { design }\end{array}$ & $\begin{array}{l}\text { Order/ } \\
\text { accessibility }\end{array}$ & $\begin{array}{l}\text { Field study: Hospital } \\
\text { cafeteria }\end{array}$ & USA (Boston) & $\begin{array}{l}4642 \text { employees of a hospital } \\
\text { cafeteria ( } 71 \% \text { females) }\end{array}$ & 41 & N/A \\
\hline Meyers et al., 1980 & $\begin{array}{l}\text { Experiment (repeated } \\
\text { measures possible) }\end{array}$ & $\begin{array}{l}\text { Order/ } \\
\text { accessibility }\end{array}$ & $\begin{array}{l}\text { Field study: hospital } \\
\text { cafeteria }\end{array}$ & USA (Memphis) & $\begin{array}{l}\text { Customers in hospital } \\
\text { cafeteria. } 4412 \\
\text { observations. Separate } \\
\text { analysis for normal-weight, } \\
\text { overweight and obese } \\
\text { subjects }\end{array}$ & N/A (adults) & $\begin{array}{l}\text { Normal weight and } \\
\text { overweight, assessed } \\
\text { by observer }\end{array}$ \\
\hline
\end{tabular}


It was not possible to quantify and directly compare the effect sizes of the included studies, as the study designs were too variable. Most of the studies were randomised-controlled experiments, and only one study used correlation analysis to study the relationship between distance and snack selection ${ }^{(28)}$. This study found that the distance from the serving bowl significantly predicted the number of crackers and carrot slices consumed by children ${ }^{(28)}$.

Between-subject experiments were the most common study design, whereas within-subject, repeated-measures designs were rarely used. Only one study used a longitudinal design ${ }^{(33)}$, which was a follow-up assessment of the intervention described by Thorndike et al. $^{(34)}$ and was based on the same choice architecture intervention. Both of these studies were retained in the review because they had assessed different outcome measures and were complementary.

Most studies assessed food selection or choice probability using $\chi^{2}$ tests, whereas only a few studies objectively measured actual food intake in terms of food weight (g) or energy $(\mathrm{kJ} / \mathrm{kcal})$ content. The intervention description and findings of the included studies are summarised in Table 2.

\section{Discussion}

Out of eighteen studies where food position or order was manipulated, sixteen showed a positive effect on food choice, meaning that the participants were nudged towards a more healthy food choice. In the two experiments ${ }^{(32)}$ where positional changes had no impact on food choice, the degree of manipulation was only a minor change in position, with all the foods remaining within reach. This indicates that the strength of the effect appears to depend on the type of positional manipulation (order $v$. distance), as well as the magnitude of the change, or how far away foods are placed.

Only one study assessed compensatory food choices ${ }^{(35)}$, showing that changes in position resulted in compensatory choices within same food categories. Further, movement of potato chips to a more distant location, and hence a reduction in chips selection, was accompanied by an increase in starch selection choices among the foods that still remained proximal $^{(35)}$. For portion size changes, there is some evidence from previous research that reducing offered portion sizes does not result in immediate compensation ${ }^{(36)}$. However, in that particular study, the intervention was conscious, and consumers' self-control was activated by having servers ask customers in a fast food restaurant whether they wanted to downsize portions. Other studies, in which the overall energy of a meal bundle for children was reduced, without the participants being aware, found that the overall energy intake was significantly reduced ${ }^{(37)}$. More research on compensatory behaviours is required to implement effective interventions in practice.

The overall quality of the included studies was neutral. Only a few papers described the procedures sufficiently well to allow a clear evaluation of all validity questions. In particular, the questions that related to subject selection, recruitment procedures and comparison of study groups were unclear or not 


\section{N British Journal of Nutrition}

Table 2. Intervention description and findings of the included studies ( $n$ 18)

\begin{tabular}{|c|c|c|c|c|c|c|c|c|c|}
\hline Author, year & $\begin{array}{l}\text { Description of } \\
\text { intervention }\end{array}$ & $\begin{array}{l}\text { Context (setting and } \\
\text { participants) }\end{array}$ & $\begin{array}{l}\text { Magnitude of change in } \\
\text { effort }\end{array}$ & Type of food involved & $\begin{array}{l}\text { Data analysis } \\
\text { method }\end{array}$ & $\begin{array}{l}\text { Dependent variables } \\
\text { (unit) }\end{array}$ & Magnitude of the effect & Main finding & Conclusions \\
\hline $\begin{array}{l}\text { Engell et al., } \\
\qquad 1996\end{array}$ & $\begin{array}{l}\text { Water pitcher on table, } v \text {. } \\
\text { dispenser at } 20 \mathrm{ft} \text { or } \\
40 \mathrm{ft} \text { distance }\end{array}$ & $\begin{array}{l}\text { Customers in Army } \\
\text { research centre } \\
\text { dining hall }\end{array}$ & $\begin{array}{l}\text { Major variation in } \\
\text { proximity. Large } \\
\text { increase in effort to } \\
\text { obtain water at a } \\
\text { dispenser across } \\
\text { room or in another } \\
\text { room }\end{array}$ & Water & ANOVA & $\begin{array}{l}\text { Water } \\
\quad \text { consumption (g) }\end{array}$ & $\begin{array}{l}\text { Significant main effect of } \\
\text { proximity on intake: } \\
F_{2,33}=8.4, P<0.001, \\
\text { post hoc tests: } \\
\text { significant reduction for } \\
\text { distant conditions } \\
\text { compared with } \\
\text { proximate condition, no } \\
\text { difference between the } \\
\text { two more distant } \\
\text { conditions }\end{array}$ & $\begin{array}{l}\text { Major reduction of water } \\
\text { intake if dispenser is } \\
\text { further away (on table } \\
v .20 \text { or } 40 \text { feet). No } \\
\text { difference between } 20 \\
\text { feet and } 40 \text { feet. No } \\
\text { effect on other food } \\
\text { intake }\end{array}$ & $\begin{array}{l}\text { Effort to obtain water } \\
\text { determined } \\
\text { amount } \\
\text { consumed }\end{array}$ \\
\hline $\begin{array}{l}\text { Maas et al., } \\
2012 \\
\text { (study 1) }\end{array}$ & $\begin{array}{l}\text { Distance to snack bowl } \\
\text { was varied at } 20,70 \\
\text { and } 140 \mathrm{~cm}\end{array}$ & $\begin{array}{l}\text { Staff/students } \\
\text { recruited to } \\
\text { laboratory on } \\
\text { university campus }\end{array}$ & $\begin{array}{l}\text { Medium: } 70 \text { and } 140 \mathrm{~cm} \\
\text { proximity variation } \\
\text { required standing up }\end{array}$ & $\begin{array}{l}\text { Candy: Chocolate } \\
\text { M\&M's (without } \\
\text { peanuts) } 1 \mathrm{~kg}\end{array}$ & $\begin{array}{l}\text { Logistic regression, } \\
\text { ANCOVA (control } \\
\text { for chocolate } \\
\text { liking) }\end{array}$ & $\begin{array}{l}\text { Amount of snack } \\
\text { consumed }(\mathrm{g}) \text { and } \\
\text { risk of } \\
\text { compensatory } \\
\text { behaviour }\end{array}$ & $\begin{array}{l}\text { Significant main effect of } \\
\text { proximity on intake: } \\
F_{2,73}=7.59, P=0.001 \\
\text { Post hoc tests: } \\
\text { significant reduction for } \\
\text { distant conditions } \\
\text { compared with } \\
\text { proximate condition, no } \\
\text { difference between the } \\
\text { two more distant } \\
\text { conditions }\end{array}$ & $\begin{array}{l}\text { An increase in distance } \\
\text { had a significant effect } \\
\text { on the probability of } \\
\text { snack consumption } \\
\text { even for an increase } \\
\text { from } 20 \text { to } 70 \mathrm{~cm} \text {. } \\
\text { No effect for } \\
\text { compensatory eating } \\
\text { was found }\end{array}$ & $\begin{array}{l}\text { Distance affected } \\
\text { intake, but } \\
\text { salience did not }\end{array}$ \\
\hline $\begin{array}{l}\text { Maas et al., } \\
2012\end{array}$ & $\begin{array}{l}\text { Distance to snack bowl } \\
\text { was varied at } 20,70 \\
\text { and } 140 \mathrm{~cm}\end{array}$ & $\begin{array}{l}\text { Staff/students } \\
\text { recruited to } \\
\text { laboratory on } \\
\text { university campus }\end{array}$ & $\begin{array}{l}\text { Medium: } 20,70 \text { and } \\
140 \mathrm{~cm} .70 \text { and } \\
140 \mathrm{~cm} \text { required } \\
\text { standing up }\end{array}$ & $\begin{array}{l}\text { Candy: Chocolate } \\
\text { M\&M's (without } \\
\text { peanuts) } 1 \mathrm{~kg}\end{array}$ & ANOVA & $\begin{array}{l}\text { Amount of snack } \\
\text { consumed (g), } \\
\text { perception of } \\
\text { salience and } \\
\text { effort, likelihood of } \\
\text { consumption }\end{array}$ & $\begin{array}{l}\text { Significant main effect of } \\
\text { proximity on intake: } \\
F_{2,51}=3 \cdot 8, P=0.029, \\
\text { post hoc tests: } \\
\text { significant reduction for } \\
\text { distant conditions } \\
\text { compared with } \\
\text { proximate condition, no } \\
\text { difference between the } \\
\text { two more distant } \\
\text { conditions }\end{array}$ & $\begin{array}{l}\text { An increase in distance } \\
\text { had a significant effect } \\
\text { on the probability of } \\
\text { snack consumption } \\
\text { even for an increase } \\
\text { from } 20 \text { to } 70 \mathrm{~cm} \text {. } \\
\text { Perceived effort } \\
\text { increased in distant } \\
\text { conditions but not } \\
\text { salience }\end{array}$ & $\begin{array}{l}\text { Significant effect of } \\
\text { proximity on } \\
\text { intake. Perceived } \\
\text { effort was higher } \\
\text { in the two distant } \\
\text { conditions, but not } \\
\text { perceived } \\
\text { salience }\end{array}$ \\
\hline $\begin{array}{l}\text { Meiselman } \\
\text { et al., 1994 } \\
\text { (study 1) }\end{array}$ & $\begin{array}{l}\text { Move candy from cash } \\
\text { point to distant snack } \\
\text { bar }\end{array}$ & $\begin{array}{l}\text { Customers } \\
\text { (students) of } \\
\text { university } \\
\text { cafeteria }\end{array}$ & $\begin{array}{l}\text { Major; increase of } \\
\text { distance }(20 \mathrm{~m}) \text { and } \\
\text { waiting at separate } \\
\text { queue. } \\
\text { Plus reduction in } \\
\text { availabilitity, (from four } \\
\text { cash registers to one } \\
\text { snack bar) }\end{array}$ & $\begin{array}{l}9 \text { food categories: } \\
\text { main dishes: } \\
\text { pizza, } \\
\text { alternatives, } \\
\text { salads, sandwich; } \\
\text { desserts: fruit, } \\
\text { accessory foods, } \\
\text { candy: chocolate, } \\
\text { chocolate } \\
\text { containing bars } \\
\text { and muesli bars }\end{array}$ & Binominal model $\left(\chi^{2}\right)$ & $\begin{array}{l}\text { Candy selection with } \\
\text { meals (selection } \\
\text { rates) }\end{array}$ & $\begin{array}{l}\text { Less candy selected in } \\
\text { nudging condition: } \\
\chi^{2}(1)=17.78, P<0.001 . \\
\text { Trend towards more } \\
\text { total desserts: } \\
\chi^{2}(1)=2 \cdot 21, P<0.1 \\
\text { (ns), no effect on other } \\
\text { foods }\end{array}$ & $\begin{array}{l}\text { Less candy was } \\
\text { purchased during the } \\
\text { intervention week. } \\
\text { However, participants } \\
\text { who chose candy in } \\
\text { the first week chose } \\
\text { more dessert fruit or } \\
\text { accessory foods } \\
\text { during the intervention } \\
\text { week }\end{array}$ & $\begin{array}{l}\text { Major increase in } \\
\text { effort to obtain an } \\
\text { unhealthy food } \\
\text { can reduce the } \\
\text { consumption of } \\
\text { the food. People } \\
\text { may partially } \\
\text { compensate } \\
\text { unhealthy choices }\end{array}$ \\
\hline $\begin{array}{l}\text { Meiselman } \\
\text { et al., 1994 } \\
\text { (study 2) }\end{array}$ & $\begin{array}{l}\text { Move potato chips from } \\
\text { cash register to } \\
\text { distant snack bar }\end{array}$ & $\begin{array}{l}\text { Customers } \\
\text { (students) of } \\
\text { university } \\
\text { cafeteria }\end{array}$ & $\begin{array}{l}\text { Major; increase of } \\
\text { distance }(20 \mathrm{~m}) \text { and } \\
\text { waiting at separate } \\
\text { queue. } \\
\text { Plus reduction in } \\
\text { availability, (from four } \\
\text { cash registers to one } \\
\text { snack bar) }\end{array}$ & $\begin{array}{l}11 \text { food categories: } \\
\text { main meal: } \\
\text { pizzas, starch, } \\
\text { vegetables, } \\
\text { salads, bread, } \\
\text { sandwiches, } \\
\text { dessert, fruit, } \\
\text { crisps, sweets/ } \\
\text { cakes, sauces, } \\
\text { candy, drinks }\end{array}$ & $\begin{array}{l}\text { Fleiss's formula and } \\
\chi^{2} \text { tests }\end{array}$ & $\begin{array}{l}\text { Potato chips } \\
\text { selection with } \\
\text { meals (selection } \\
\text { rates) }\end{array}$ & $\begin{array}{l}\text { Less potato chips selected } \\
\text { in nudging condition: } \\
\chi^{2}(1)=77.27, P<0.001 . \\
\text { More starch foods } \\
\text { during intervention: } \\
\chi^{2}(1)=6.20, P<0.001\end{array}$ & $\begin{array}{l}\text { Increased effort reduced } \\
\text { potato chips selection, } \\
\text { reduction was } \\
\text { accompanied by } \\
\text { increased starch } \\
\text { selection }\end{array}$ & $\begin{array}{l}\text { Varying effort can } \\
\text { increase or } \\
\text { decrease } \\
\text { consumption. } \\
\text { Foods are } \\
\text { substituted with } \\
\text { other foods (within } \\
\text { same food group) }\end{array}$ \\
\hline $\begin{array}{l}\text { Musher- } \\
\text { Eizenman } \\
\text { et al., } 2010\end{array}$ & $\begin{array}{l}\text { Children were randomly } \\
\text { placed at varying } \\
\text { distances to healthy } \\
\text { and unhealthy snacks }\end{array}$ & Children in day care & $\begin{array}{l}\text { Major: children had to } \\
\text { stand up and come up } \\
\text { to the experimenter } \\
\text { from varying distances } \\
\text { and ask for more } \\
\text { snack }\end{array}$ & $\begin{array}{l}\text { Snacks: high energy } \\
\text { dense animal } \\
\text { crackers } v \text {. carrot } \\
\text { slices }\end{array}$ & $\begin{array}{l}\text { Hierarchical } \\
\text { regression }\end{array}$ & $\begin{array}{l}\text { Consumption of } \\
\text { crackers and } \\
\text { carrot slices } \\
\text { (number of pieces } \\
\text { consumed) }\end{array}$ & $\begin{array}{l}\text { Distance from serving bowl } \\
\text { predicted intake: } \\
\text { Distance from crackers: } \\
\beta=-0.41, P<0.05 \\
\left(\Delta R^{2}=0.17\right) \\
\text { Distance from carrots } \\
\beta=-0.38, P<0.05 \\
\left(\Delta R^{2}=0.14\right)\end{array}$ & $\begin{array}{l}\text { Distance from the } \\
\text { serving bowl } \\
\text { significantly predicted } \\
\text { number of crackers } \\
\text { and carrot slices } \\
\text { consumed }\end{array}$ & $\begin{array}{l}\text { Proximity influences } \\
\text { consumption of } \\
\text { healthy and } \\
\text { unhealthy snacks } \\
\text { in children }\end{array}$ \\
\hline
\end{tabular}


Table 2. Continued

\begin{tabular}{|c|c|c|c|c|c|c|c|c|c|}
\hline Author, year & $\begin{array}{l}\text { Description of } \\
\text { intervention }\end{array}$ & $\begin{array}{l}\text { Context (setting and } \\
\text { participants) }\end{array}$ & $\begin{array}{l}\text { Magnitude of change in } \\
\text { effort }\end{array}$ & Type of food involved & $\begin{array}{l}\text { Data analysis } \\
\text { method }\end{array}$ & $\begin{array}{l}\text { Dependent variables } \\
\text { (unit) }\end{array}$ & Magnitude of the effect & Main finding & Conclusions \\
\hline $\begin{array}{l}\text { Privitera et al., } \\
2012\end{array}$ & $\begin{array}{l}\text { Manipulation of proximity } \\
\text { (near } v \text {. far) and } \\
\text { visibility (clear } v \text {. } \\
\text { opaque bowl) of } \\
\text { healthy foods }\end{array}$ & $\begin{array}{l}\text { Students recruited to } \\
\text { laboratory on } \\
\text { university campus }\end{array}$ & $\begin{array}{l}\text { Medium: serving bowl } \\
\text { placed } 2 \mathrm{~m} \text { away on } \\
\text { counter or on table } \\
\text { within arm's reach }\end{array}$ & $\begin{array}{l}\text { Snacks (healthy): } \\
\text { fruits and } \\
\text { vegetables }\end{array}$ & ANOVA & $\begin{array}{l}\text { Apple and carrot } \\
\text { consumption } \\
\text { (number of pieces } \\
\text { consumed) }\end{array}$ & $\begin{array}{l}\text { Significant effect of } \\
\text { distance on intake: } \\
\text { Apple: } F_{1,44}=25.46, \\
P<0.001 ; \\
\text { Carrots: } \\
P<0.04\end{array}$ & $\begin{array}{l}\text { Proximity increased } \\
\text { intake of both, fruit } \\
\text { and vegetable intake } \\
\text { (visibility only affected } \\
\text { of fruit intake) }\end{array}$ & $\begin{array}{l}\text { Proximate can } \\
\text { increase } \\
\text { consumption of } \\
\text { healthy foods. } \\
\text { The effect was } \\
\text { stronger for } \\
\text { apples compared } \\
\text { to carrots. This } \\
\text { might be because } \\
\text { fruits are sweeter } \\
\text { and more } \\
\text { appealing than } \\
\text { vegetables }\end{array}$ \\
\hline $\begin{array}{l}\text { Privitera et al., } \\
2014\end{array}$ & $\begin{array}{l}\text { Effect of proximity was } \\
\text { tested in a competitive } \\
\text { food environment with } \\
\text { healthy food and } \\
\text { unhealthy food at } \\
\text { different distances }\end{array}$ & $\begin{array}{l}\text { Students recruited to } \\
\text { laboratory on } \\
\text { university campus }\end{array}$ & $\begin{array}{l}\text { Medium: } 2 \mathrm{~m} \mathrm{v} \text {. arms } \\
\text { reach }\end{array}$ & $\begin{array}{l}\text { Snacks: apple slices } \\
\text { (healthy food) } v . \\
\text { buttered popcorn } \\
\text { (high-fat } \\
\text { unhealthy food) }\end{array}$ & $\begin{array}{l}\text { ANCOVA: BMI as } \\
\text { covariate }\end{array}$ & $\begin{array}{l}\text { Apple and popcorn } \\
\text { consumption (kcal } \\
\text { and proportion) }\end{array}$ & 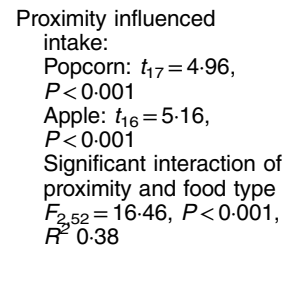 & $\begin{array}{l}\text { The food that was placed } \\
\text { closer to the } \\
\text { participants was } \\
\text { consumed most, } \\
\text { regardless of } \\
\text { preference }\end{array}$ & $\begin{array}{l}\text { Making a low-energy } \\
\text { food more } \\
\text { proximate than a } \\
\text { high-energy food, } \\
\text { will reduce total } \\
\text { energy intake, } \\
\text { even if a high- } \\
\text { energy and more } \\
\text { preferred food is } \\
\text { also avaiable but } \\
\text { less proximate }\end{array}$ \\
\hline $\begin{array}{l}\text { Wansink } \\
\quad \text { et al., } 2006\end{array}$ & $\begin{array}{l}\text { Manipulation of proximity } \\
\text { (near v. far) and } \\
\text { visibility (clear } v \text {. } \\
\text { opaque bowl) of candy }\end{array}$ & $\begin{array}{l}\text { Female staff within } \\
\text { their offices at } \\
\text { university }\end{array}$ & $\begin{array}{l}\text { Medium: } 2 \mathrm{~m} \mathrm{v} \text {. arms } \\
\text { reach }\end{array}$ & $\begin{array}{l}\text { Candy (individually } \\
\text { wrapped } \\
\text { chocolates) }\end{array}$ & $\begin{array}{l}\text { ANOVA (post hoc } \\
t \text { tests) }\end{array}$ & $\begin{array}{l}\text { Chocolate } \\
\text { consumption } \\
\text { (number of } \\
\text { pieces) }\end{array}$ & $\begin{array}{l}1.8 \text { chocolates more } \\
\text { consumed if they were } \\
\text { proximate. Effect size } \\
\text { unclear }\end{array}$ & $\begin{array}{l}\text { More candy consumed if } \\
\text { it is more proximate }\end{array}$ & $\begin{array}{l}\text { Proximity increases } \\
\text { consumption. } \\
\text { People } \\
\text { overestimate } \\
\text { consumption of } \\
\text { less proximate } \\
\text { foods }\end{array}$ \\
\hline $\begin{array}{l}\text { Keller \& } \\
\quad \text { Bucher, } \\
2014\end{array}$ & $\begin{array}{l}\text { Manipulation of snack } \\
\text { bar order on tray; } \\
\text { healthy bar at the side } \\
v \text {. in the middle of an } \\
\text { assortment }\end{array}$ & $\begin{array}{l}\text { Students recruited } \\
\text { on campus at } \\
\text { university }\end{array}$ & $\begin{array}{l}\text { Minor or none: only } \\
\text { positions of foods } \\
\text { within reach were } \\
\text { altered }\end{array}$ & $\begin{array}{l}\text { Snacks: healthy } \\
\text { apple cereal bar } \\
v . \text { unhealthy } \\
\text { cereal bars } \\
\text { (chocolate cereal } \\
\text { bars) }\end{array}$ & $\chi^{2}$ & $\begin{array}{l}\text { Cereal bar choice } \\
\text { (selection rates) }\end{array}$ & $\begin{array}{l}\text { Significant influence of } \\
\text { position on selection: } \\
\chi^{2}(2)=14.95, P<0.001\end{array}$ & $\begin{array}{l}\text { The healthy bar was } \\
\text { selected more often, } \\
\text { when it was placed in } \\
\text { the middle }\end{array}$ & $\begin{array}{l}\text { Changing the } \\
\text { position of snacks } \\
\text { can nudge } \\
\text { healthier choices }\end{array}$ \\
\hline Levitz, 1976 & $\begin{array}{l}\text { Order of desserts with } \\
\text { varying energy } \\
\text { content in within } \\
\text { shelves; front } v \text {. rear } \\
\text { position }\end{array}$ & $\begin{array}{l}\text { Customers in } \\
\text { hospital cafeteria }\end{array}$ & $\begin{array}{l}\text { Minor: change within } \\
\text { display }\end{array}$ & $\begin{array}{l}\text { Three types of } \\
\text { dessert; } \\
\text { High energy: } \\
\text { cakes and pies, } \\
1464 \mathrm{~kJ} / \mathrm{serving} \\
\text { (350 kcal/serving) } \\
\text { Low-energy: fruit, } \\
\text { gelatine, } 314 \mathrm{~kJ} / \\
\text { serving ( } 75 \mathrm{kcal} / \\
\text { serving) } \\
\text { Moderate: } \\
\text { custard, pudding }\end{array}$ & $x^{2}$ & $\begin{array}{l}\text { Dessert sales } \\
\quad \text { (selection rates) }\end{array}$ & $\begin{array}{l}\text { Normal-weight subjects: } \\
\text { low-energy dessert } \\
\text { more available: } \\
\chi^{2}=4 \cdot 13, P<0.05 \\
\text { High-energy dessert } \\
\text { more available: } \\
\chi^{2}=3.96, P<0.05 \\
\text { Obese subjects: low- } \\
\text { energy dessert more } \\
\text { available: } \chi^{2}=17.67, \\
P<0.05 \\
\text { High-energy dessert } \\
\text { more available: ns }\end{array}$ & $\begin{array}{l}\text { Normal-weight } \\
\text { individuals } \\
\text { consistently selected } \\
\text { the most available } \\
\text { choice } \\
\text { Obese people chose } \\
\text { more low-energy } \\
\text { dessert if it was made } \\
\text { more salient. No } \\
\text { change for obese if } \\
\text { high-energy dessert } \\
\text { was more salient }\end{array}$ & $\begin{array}{l}\text { Both, obese and } \\
\text { normal-weight } \\
\text { individuals are } \\
\text { responsive } \\
\text { changes in food } \\
\text { positioning }\end{array}$ \\
\hline $\begin{array}{l}\text { Levy et al., } \\
2012\end{array}$ & $\begin{array}{l}\text { 2-phase intervention 1st } \\
\text { phase: labelling of } \\
\text { healthy and unhealthy } \\
\text { food. 2nd phase: } \\
\text { placement variation of } \\
\text { various foods }\end{array}$ & $\begin{array}{l}\text { Customers in } \\
\text { hospital cafeteria }\end{array}$ & $\begin{array}{l}\text { Minor: for sandwiches } \\
\text { and chips: only } \\
\text { positions of foods } \\
\text { within shelves were } \\
\text { altered. Eye level } v \text {. } \\
\text { below eye level } \\
\text { position. } \\
\text { Medium for bottled } \\
\text { water: bottled water } \\
\text { available at several } \\
\text { locations in nudging } \\
\text { condition }\end{array}$ & $\begin{array}{l}\text { Beverages, } \\
\text { sandwiches, chips }\end{array}$ & $\begin{array}{l}\text { Linear regression } \\
\text { (demographics as } \\
\text { controls) }\end{array}$ & $\begin{array}{l}\text { Sales of healthy and } \\
\text { unhealthy foods } \\
\text { (percentage of } \\
\text { change) }\end{array}$ & $\begin{array}{l}\text { Decrease of red item } \\
\text { purchases by } 4.1 \% \\
\text { during the Phase } 2 \\
\text { choice architecture } \\
\text { intervention }\end{array}$ & $\begin{array}{l}\text { Repositioning red } \\
\text { (unhealthy) beverages } \\
\text { reduced sales in } \\
\text { addition to the colour } \\
\text { coding intervention }\end{array}$ & $\begin{array}{l}\text { Choice architecture } \\
\text { intervention } \\
\text { improved food } \\
\text { and beverage } \\
\text { choices among } \\
\text { employees from } \\
\text { all racial and } \\
\text { socio-economic } \\
\text { backgrounds on } \\
\text { top of the labelling } \\
\text { intervention }\end{array}$ \\
\hline
\end{tabular}


Table 2. Continued

\begin{tabular}{|c|c|c|c|c|c|c|c|c|c|}
\hline Author, year & $\begin{array}{l}\text { Description of } \\
\text { intervention }\end{array}$ & $\begin{array}{l}\text { Context (setting and } \\
\text { participants) }\end{array}$ & $\begin{array}{l}\text { Magnitude of change in } \\
\text { effort }\end{array}$ & Type of food involved & $\begin{array}{l}\text { Data analysis } \\
\text { method }\end{array}$ & $\begin{array}{l}\text { Dependent variables } \\
\text { (unit) }\end{array}$ & Magnitude of the effect & Main finding & Conclusions \\
\hline $\begin{array}{l}\text { Meyers et al., } \\
\quad 1980\end{array}$ & $\begin{array}{l}\text { Manipulation of order of } \\
\text { desserts with varying } \\
\text { energy content within } \\
\text { shelves; front } v \text {. rear } \\
\text { position }\end{array}$ & $\begin{array}{l}\text { Customers in } \\
\text { hospital cafeteria }\end{array}$ & $\begin{array}{l}\text { Minor: change within } \\
\text { display }\end{array}$ & $\begin{array}{l}\text { Desserts: two types; } \\
\text { high energy: } \\
\text { cakes and pies } \\
\text { Low energy: fresh } \\
\text { fruit and gelatine }\end{array}$ & $\begin{array}{l}\text { Multiple contingency } \\
\text { analysis }\left(x^{2}\right)\end{array}$ & $\begin{array}{l}\text { Dessert sales } \\
\quad \text { (selection rates) }\end{array}$ & $\begin{array}{l}\text { Likelihood to choose a } \\
\text { dessert in front was } \\
\text { increased. } \\
\chi^{2}(2)=22 \cdot 3, P<0.001 \\
\text { (significant interaction } \\
\text { between dessert array } \\
\text { and dessert choice) }\end{array}$ & $\begin{array}{l}\text { Subjects were more likely } \\
\text { to choose the dessert } \\
\text { in front. No difference } \\
\text { between overweight } \\
\text { and normal-weight } \\
\text { subjects }\end{array}$ & $\begin{array}{l}\text { All subjects were } \\
\text { more likely to } \\
\text { select the dessert } \\
\text { in front }\end{array}$ \\
\hline $\begin{array}{l}\text { Rozin et al., } \\
2013 \\
\text { (study 3) }\end{array}$ & $\begin{array}{l}\text { Manipulation of salad } \\
\text { order at self-service } \\
\text { salad bar: less } \\
\text { accessible middle } \\
\text { position } v \text {. more } \\
\text { accessible edge } \\
\text { position }\end{array}$ & $\begin{array}{l}\text { Customers in } \\
\text { hospital cafeteria }\end{array}$ & $\begin{array}{l}\text { Minor: change within } \\
\text { display }\end{array}$ & $\begin{array}{l}8 \text { ingredients at a } \\
\text { salad bar: } \\
\text { chicken, egg, } \\
\text { tuna, salmon, } \\
\text { tomatoes, carrots, } \\
\text { mushrooms } \\
\text { cucumbers }\end{array}$ & Multiple $t$ tests & $\begin{array}{l}\text { Sales (weight) from } \\
\text { pay-by weight } \\
\text { salad bar }\end{array}$ & $\begin{array}{l}\text { Average sales of each } \\
\text { ingredient was reduced } \\
\text { by } 8.9 \% \text { in the middle } \\
\text { position compared with } \\
\text { the edge position } \\
\left(t_{7}=-4.13, P<0.01,\right. \\
Z \text {-score }=0.30)\end{array}$ & $\begin{array}{l}\text { Sales of each of the eight } \\
\text { ingredients diminished } \\
\text { when displayed in the } \\
\text { less accessible middle } \\
\text { row }\end{array}$ & $\begin{array}{l}\text { Food positions at } \\
\text { self-serving pay- } \\
\text { by-weight salad } \\
\text { bar had a } \\
\text { significant } \\
\text { influence on sales }\end{array}$ \\
\hline $\begin{array}{l}\text { Thorndike } \\
\text { et al., } 2012\end{array}$ & See Levy et al..$^{(33)}$ & $\begin{array}{l}\text { Customers in } \\
\text { hospital cafeteria }\end{array}$ & $\begin{array}{l}\text { Medium and minor; see } \\
\text { Levy et al. }{ }^{(33)}\end{array}$ & $\begin{array}{l}\text { Beverages, } \\
\text { sandwiches, chips }\end{array}$ & Logistic regression & $\begin{array}{l}\text { Sales of healthy and } \\
\text { unhealthy foods, } \\
\text { selection rates }\end{array}$ & $\begin{array}{l}\text { Decrease of unhealthy } \\
\text { beverage purchase by } \\
11.4 \% \text {. } \\
\text { Increase of healthy } \\
\text { beverage purchase by } \\
4 \% \text {. } \\
\text { Increased sales of } \\
\text { bottled water by } 25 \% \text {, } \\
P>0.001\end{array}$ & $\begin{array}{l}\text { Small but significant } \\
\text { increases in sales by } \\
\text { reordering } \\
\text { sandwiches and chips } \\
\text { on shelves }\end{array}$ & $\begin{array}{l}\text { Choice architecture } \\
\text { intervention } \\
\text { improved food } \\
\text { and beverage } \\
\text { choices }\end{array}$ \\
\hline $\begin{array}{l}\text { van Kleef } \\
\quad \text { et al., 2012 } \\
\text { (study 1) }\end{array}$ & $\begin{array}{l}\text { Manipulation of snack } \\
\text { position (healthy foods } \\
\text { on top v. at bottom) }\end{array}$ & $\begin{array}{l}\text { Undergraduate } \\
\text { students recruited } \\
\text { to laboratory on } \\
\text { university campus }\end{array}$ & $\begin{array}{l}\text { Minor; only positions of } \\
\text { foods on screen were } \\
\text { altered }\end{array}$ & $\begin{array}{l}\text { Snacks: an } \\
\text { assortment of } 16 \\
\text { (out of 24) healthy } \\
\text { and unhealthy } \\
\text { snacks; fresh and } \\
\text { dried fruit and } \\
\text { vegetables, } \\
\text { savoury and salty } \\
\text { snacks, and } \\
\text { sweet biscuits and } \\
\text { chocolates }\end{array}$ & $\begin{array}{l}\text { Logistic regression } \\
\text { and ANOVA }\end{array}$ & $\begin{array}{l}\text { Snack choice on } \\
\text { screen }\end{array}$ & $\begin{array}{l}\text { No significant differences } \\
\text { were observed in the } \\
\text { 'healthy snacks at the } \\
\text { top' conditions (30.38\% } \\
\text { choose healthy) } \\
\text { compared with the } \\
\text { bottom conditions } \\
(27.85 \% ;(1, n 158)= \\
1.29, P=0.34)\end{array}$ & $\begin{array}{l}\text { No significant effect of } \\
\text { shelf position on } \\
\text { snack choice }\end{array}$ & $\begin{array}{l}\text { Field study showed a } \\
\text { trend that } \\
\text { consumption of } \\
\text { healthy foods was } \\
\text { affected, but that } \\
\text { consumption of } \\
\text { unhealthy foods } \\
\text { was not altered }\end{array}$ \\
\hline $\begin{array}{l}\text { van Kleef } \\
\text { et al., 2012 } \\
\text { (study 2) }\end{array}$ & $\begin{array}{l}\text { Manipulation of shelf } \\
\text { position (healthy foods } \\
\text { on top } v \text {. at bottom) }\end{array}$ & $\begin{array}{l}\text { Customers in } \\
\text { hospital cafeteria }\end{array}$ & $\begin{array}{l}\text { Medium; positions of } \\
\text { foods within a shelf } \\
\text { were altered, reaching } \\
\text { some foods required } \\
\text { bending down }\end{array}$ & $\begin{array}{l}\text { Snacks: an } \\
\text { assortment of } 16 \\
\text { healthy and } \\
\text { unhealthy snacks: } \\
\text { fresh and dried } \\
\text { fruit and } \\
\text { vegetables, } \\
\text { savoury and salty } \\
\text { snacks, and } \\
\text { sweet biscuits and } \\
\text { chocolates }\end{array}$ & ANOVA & Snack sales & $\begin{array}{l}\text { No significant effect of } \\
\text { shelf arrangement on } \\
\text { total snack sales } \\
\left(F_{1,6}=3 \cdot 84, P=0 \cdot 1 \text {. }\right. \\
\text { Separate analysis for } \\
\text { healthy and unhealthy } \\
\text { snacks revealed that no } \\
\text { effect on unhealthy } \\
\text { items but a trend } \\
\text { towards higher sales of } \\
\text { healthy items when } \\
\text { healthy foods were } \\
\text { placed on top } \\
\left(F_{1,6}=5.03, P=0.07\right.\end{array}$ & $\begin{array}{l}\text { No significant effect of } \\
\text { shelf position for } \\
\text { unhealthy foods, but a } \\
\text { trend to higher sales } \\
\text { for healthy foods, if } \\
\text { they were placed } \\
\text { more prominent }\end{array}$ & $\begin{array}{l}\text { No effect of } \\
\text { repositioning on } \\
\text { choice }\end{array}$ \\
\hline $\begin{array}{l}\text { Wansink } \\
\text { et al., } 2013\end{array}$ & $\begin{array}{l}\text { Food order inverted at } \\
\text { breakfast buffet: } \\
\text { healthiest to least } \\
\text { healthy v. least healthy } \\
\text { to healthiest food }\end{array}$ & $\begin{array}{l}\text { Conference } \\
\text { participants at } \\
\text { conference venue }\end{array}$ & $\begin{array}{l}\text { Medium; order of foods } \\
\text { on buffet was altered }\end{array}$ & $\begin{array}{l}7 \text { item buffet: cheesy } \\
\text { eggs, potatoes, } \\
\text { bacon, cinnamon } \\
\text { roll, low-fat } \\
\text { granola, low-fat } \\
\text { yogurt and fruit }\end{array}$ & $\chi^{2}$, Maxwell tests & $\begin{array}{l}\text { Breakfast item } \\
\text { selection } \\
\text { (selection rates) }\end{array}$ & $\begin{array}{l}\text { Significant effect of order } \\
\text { on choice: } \chi^{2}(6)=25 \cdot 1 \\
P<0.001, \text { Stuart- } \\
\text { Maxwell test }=171 \cdot 2 \\
(P<0.001, \text { df }=6)\end{array}$ & $\begin{array}{l}\text { Order significantly } \\
\text { influences what } \\
\text { people select }\end{array}$ & $\begin{array}{l}\text { First foods in line } \\
\text { were consumed } \\
\text { most often }\end{array}$ \\
\hline
\end{tabular}


applicable. Studies were classified as unclear or not being free from bias because of the use of cash incentives or course credit being offered to participants. This may be an artifact of the naturalistic setting of the studies, such as universities and workplace canteens.

There is a lack of research investigating long-term outcomes of positional interventions, and it is not clear whether changes in product order or distance would have sustained effects. Specifically, it is unclear whether a potentially positive effect of a position change, such as placing healthy foods in obvious positions and very close to cafeteria check-out lines, would potentially diminish over time and that customers would return to selecting a favoured unhealthy snack. To investigate this, more studies have to be conducted that evaluate this. Changes in choice need to be assessed at different time points, ideally over several weeks and months - for example, using data from a customer loyalty card scheme to determine sustainability of the intervention.

Furthermore, only one study ${ }^{(38)}$ assessed the effect of potential covariates such as food preferences, restrained or disinhibited eating styles or health consciousness on the outcomes of position choice architecture interventions. It therefore remains unclear which individuals are susceptible to nudges. Further insight on these covariates, as well as potential influences of habit strength, is required to design effective interventions.

A reason for these data not being reported may be that it is important to ensure participants are not aware of the nudging intervention, and this is likely to be the reason most field studies did not collect this information from participants. A method that could be used to address this limitation in future research would be to implement interventions within settings where customer loyalty cards are used to collect additional data on participants' actual purchases. For this purpose, collaborations with industry or supermarket chains could be effective. This would also have the advantage that potential product price and positioning interactions could be assessed.

Previous literature suggests that nudges could be inexpensive approaches to positively impact behaviours ${ }^{(15)}$. In the studies included in this review, however, there were no calculations on potential costs and benefits. Factual data on previously hypothesised benefits are required to make effective recommendations for policymakers.

Only two studies differentiated between healthy and overweight consumers and whether positional interventions were different based on body weight ${ }^{(27,31)}$. They both concluded that the positional nudges were effective irrespective of weight status. Further, one study assessed socio-economic status and reported that it had no influence on whether positional interventions were effective ${ }^{(33)}$. These findings concur with previous literature, which suggest that nudging effects work via subconscious mechanisms, and therefore have equal impact regardless of weight and socio-economic status ${ }^{(39)}$

Food position can be manipulated by changing the order of food products or by changing the distance between the food and the consumer. Both of these nudges operate in different ways. The mediating factor for the effect of distance on choice is thought to be effort, whereas for change in order it is reported to be salience ${ }^{(38)}$. Changes in order normally constitute only a minor change in effort, whereas changes in distance affect the effort required in order to obtain a food at various levels. However, more research is needed to evaluate these two aspects in detail. Future research should also clearly distinguish between studies that examine nudging in terms of food order $v$. food proximity or distance.

To date, very little is known about why positional nudges could be effective, and, in particular, it remains unclear how effects are moderated. The dual-process model ${ }^{(40)}$ states that human behaviour largely results as a function of two interacting systems: the reflective system, which generates decisions based on knowledge about facts and values, and the impulsive system, which elicits behaviour through affective responses. The first system requires cognitive capacity, whereas the second system requires no cognitive effort and is driven by feelings and immediate behaviours in response to the environment. Nudging is thought to operate mainly through the second, automatic system and affects all individuals equally. However, it remains to be elucidated whether and how factors such as health consciousness, habits or strong preferences for specific products interact with the effects. The research of Levy et al. ${ }^{(33)}$ suggests that once the social gradient effects are taken into consideration, there is still an effect towards the desired outcome in terms of food choices. This indicates that these interventions could be powerful and that cheap nudging interventions could potentially yield more than other elaborate expensive campaigns do. However, further research is required to explore this in detail.

It was not possible to conduct a meta-analysis of effect sizes as a wide range of outcome measures were reported across studies. Although the evidence that food position influences food choice was consistent across studies, it was not possible to evaluate the impact and effect size of these types of choice architecture interventions on actual food consumption and subsequent health outcomes. As has been advocated previously ${ }^{(6)}$, harmonised indicators are required that would allow comparability between experiments or interventions. We therefore strongly recommend the use of energy $(\mathrm{kJ} / \mathrm{kcal})$ or weight $(\mathrm{g})$ as outcome measures of changes in food selection and/or intake in future studies.

\section{Strengths and limitations}

This is the first systematic review that has assessed the influence of position interventions (proximity and order) on food choice. In addition to the terms 'nudging' and 'choice architecture', we used search terms such as 'distance' and 'position'. This strategy located many articles that were beyond the topic of interest such as access to fast food outlets, but ensured that older literature published before the terms 'choice architecture' and 'nudging' became popular were included.

For the purpose of this review, we defined nudging as any intervention that involved altering the non-economic properties or placement of objects or stimuli within microenvironments, with the intention of changing health-related behaviour (adapted from Hollands et al. ${ }^{(23)}$ ). We acknowledge that varying definitions of this term exist and that a disparate definition 
of the term might have led to the inclusion of different studies, and hence influence the conclusions drawn.

Literature investigating the effect of the assortment structure on buying behaviour within supermarkets was not identified with the present search strategy. The authors are aware that supermarket-related shopping behaviour has been extensively described in the marketing literature, and that it is one of the venues where behavioural interventions may have a socially relevant outcome ${ }^{(7,41)}$. This aspect was beyond the scope of the present study, which focused mainly on out-of-home meal service situations such as cafeterias or canteens. Factors affecting selection at the time of consumption and the time of purchase may differ in this situation.

In addition, it is relevant to note that there could be differences between nudges that aim to increase or decrease consumption, as well as between nudges that promote the choice of healthy foods $v$. nudges that discourage the consumption of unhealthy foods. As an example, it might be easier to promote the consumption of more (healthy) food, compared with discouraging the consumption of unhealthy (or preferred) food by positional changes. Studies in which the positions of unhealthy and healthy foods are simply switched are particularly problematic, as they lack a neutral control group, which would enable researchers to disentangle whether there was a potential bias in effectiveness of nudging depending on the food. In the present literature, studies that strategically investigated the efficacy of the positional intervention depending on food type are missing.

This review did not specifically consider any grey literature. Given the heterogeneity and the limited number of studies retrieved via the search strategy, it is plausible that a positive publication bias exists, although this was not assessed by the authors. It is interesting to note that the paternalistic nature of the concept of nudging has been discussed. In particular, it can be argued that a positional change that results in high effort to obtain an unhealthier food may be seen as a reduction in freedom of choice ${ }^{(42-45)}$. However, owing to the ethical nature of this discussion, it is beyond the scope of this review.

The synthesis of the study findings was undertaken in a narrative format as data aggregation was limited by the heterogeneity of the research in this field. Nevertheless, the current review identified gaps in the existing literature and where further research is needed.

\section{Recommendations for laboratory studies}

Although laboratory settings are limited, well-planned experiments could give insight into the strength of positional effects, and therefore help estimate the cost-effectiveness of choice architecture interventions in practice, particularly if repeated measures are applied. Laboratory settings allow the follow-up of the same individuals for data collection. Quantifiable outcome measures such as change in energy $(\mathrm{kJ} / \mathrm{kcal})$ or weight (g) of food selection or consumption should be used. Strong experimental evidence including estimations of the potential health benefits secondary to a reduction in energy intake or consumer weight loss over time is needed to inform policymakers in terms of implementing choice architecture interventions in public health settings.

\section{Recommendations for field experiments}

Although previous research suggested that substitution might occur within the same product category following a choice architecture intervention $^{(35)}$, a trial in the Belgian city of Ghent showed that meal choices were not compensated for later in the day ${ }^{(46)}$. Hence, future research should address the issue of compensation at the design stage and consider that compensatory behaviours could occur after a nudge intervention.

As for laboratory settings, we also strongly recommend the use of energy or grams of food selected/consumed as an objective outcome measure, to estimate effect sizes and potential health benefits.

Furthermore, insight into factors (e.g. preferences, habit strengths, health consciousness) that potentially influence the effectiveness of positional interventions could be gained by collecting more information on customers in cafeteria-style settings - for example, via a loyalty card scheme. This would further allow exploration of the sustainability (decay of effect over time or potential compensatory choices) over time in these settings.

\section{Reporting recommendations}

The eighteen studies included in this review did not consistently describe the choice architecture intervention that was being assessed - for example, whether 'the nudge' was a change in distance or in product positioning. On the other hand, the inclusion of distance in combination with food resulted in a large number of search results that were not relevant for the purpose of this study.

We suggest that standardised keywords and vocabulary could assist this field of research. Researchers should carefully consider the wording for their reports and could adopt the terminologies suggested by Hollands et al. ${ }^{(23)}$ to classify choice architecture interventions more clearly.

\section{Advice for practice (policymakers, food retailers)}

Choice architecture recommendations could support existing dietary guidelines, and thus potentially contribute to the adherence and compliance. Although more research is required to quantify the magnitude of positional influences on health outcomes, it is evident that choice architecture is important and that food retailers influence consumption by organising and displaying their products. Therefore, persons in charge of food organisation or food outlet design (e.g. workplaces) need to be aware of their responsibility to organise 'foodscapes' in an optimal way - for example, to stimulate consumption of healthy foods and to reduce the consumption of unhealthy foods that then could support healthy workplace initiatives. In practical terms, this means that low-energy, nutrient-dense products such as fruits and vegetables should be placed in easily accessible and prominent positions. This is particularly applicable in large self-serving setting such a school or work canteens or the canteens of residences for the elderly.

Policymakers could integrate choice architecture nudging measures to augment their existing policy documents as an important measure to enhance the effectiveness of healthy 
eating policies and procedures. In particular, this review provides evidence for policymakers, and specifically supports the use of positional changes as an effective manner to alter food choice in a desirable way.

Furthermore, the results of this review could be used for developing official recommendations regarding the implementation of choice architectural nudge interventions and to harmonise the indicators for evaluation of the effect. A good practice example would be to place salad at the beginning of the buffet in school canteens in countries where meals are provided at school.

\section{Conclusions}

Although the evidence that food position influences food choice is consistent, it is difficult to quantify the magnitude of impact on food choice and intake and the effect size of these choice architecture interventions on actual food consumption and subsequent health outcomes. Use of harmonised terminology and indicators would allow comparability between experiments or interventions and assist in moving this field forward.

\section{Acknowledgements}

The authors thank Debbie both for assistance with developing the search strategy and the database searches.

T. B. received a fellowship from the Swiss National Science Foundation (P2EZP1_159086) and the Swiss Foundation for Nutrition Research (SFEFS) to work on this project. C. C. is supported by an NHMRC Senior Research fellowship. F. J. A. P. C. is supported by IAPP-Marie Curie FP7/EU grant (agreement no. 612326 VeggiEAT). The funding sources had no influence on the design of the study.

T. B., N. D. V. and D. V. d. B. screened the abstracts; T. B. and M. E. R. extracted the results; and T. A. M. and H. T. performed the quality assessment. T. B. and F. J. A. P. C. jointly wrote the manuscript with critical input from C. C., H. T., M. E. R. and T. A. M.

The authors declare that there are no conflicts of interest.

\section{Supplementary material}

For supplementary material/s referred to in this article, please visit http://dx.doi.org/doi:10.1017/S0007114516001653

\section{References}

1. Hill JO, Wyatt HR, Reed GW, et al. (2003) Obesity and the environment: where do we go from here? Science 299, 853-855.

2. Mikkelsen BE (2011) Images of foodscapes: introduction to foodscape studies and their application in the study of healthy eating out-of-home environments. Perspect Public Health 131, 209-216.

3. Hill JO \& Peters JC (1998) Environmental contributions to the obesity epidemic. Science 280, 1371-1374.

4. Bamia C, Trichopoulos D, Ferrari P, et al. (2007) Dietary patterns and survival of older Europeans: the EPIC-Elderly study (European Prospective Investigation into Cancer and Nutrition). Public Health Nutr 10, 590-598.
5. Wills J \& Grunert K (2007) A review of research on consumer response to nutrition information on food labels in Europe from 2003-2006. Ann Nutr Metab 51, 37-38.

6. Perez-Cueto FJA, Aschemann-Witzel J, Shankar B, et al. (2012) Assessment of evaluations made to healthy eating policies in Europe: a review within the EATWELL Project. Public Health Nutr 15, 1489-1496.

7. Johnson EJ, Shu SB, Benedict GC, et al. (2012) Beyond nudges: tools of a choice architecture. Mark Lett 23, 487-504.

8. List JA \& Samek AS (2015) The behavioralist as nutritionist: leveraging behavioral economics to improve child food choice and consumption. J Health Econ 39, 135-146.

9. Riebl SK, Estabrooks PA, Dunsmore JC, et al. (2015) A systematic literature review and meta-analysis: the theory of planned behavior's application to understand and predict nutrition-related behaviors in youth. Eat Behav 18, 160-178.

10. Brambila-Macias J, Shankar B, Capacci S, et al. (2011) Policy interventions to promote healthy eating: a review of what works, what does not, and what is promising. Food Nutr Bull 32, 365-375.

11. Capacci S, Mazzocchi M, Shankar B, et al. (2012) Policies to promote healthy eating in Europe: a structured review of policies and their effectiveness. Nutr Rev 70, 188-200.

12. Hansen PG \& Jespersen A (2013) Nudge and the manipulation of choice. A framework for the responsible use of nudge approach to behaviour change in public policy. Eur J Risk Regul 1, 3-28.

13. Rozin P, Scott S, Dingley M, et al. (2011) Nudge to nobesity I: minor changes in accessibility decrease food intake. Judgm Decis Mak 6, 323-332.

14. Wansink B (2007) Mindless Eating: Why We Eat More Than We Think. New York, NY: Bantam.

15. Thaler RH \& Sunstein CR (2008) Nudge Improving Decisions About Health, Wealth and Happiness. New Haven, CT: Yale University Press.

16. House of Lords (2011) Behaviour Change. Science and Technology Select Committee. 2nd Report of Session 2010-12. London: The Authority of the House of Lords.

17. Skov LR, Lourenco S, Hansen GL, et al. (2012) Choice architecture as a means to change eating behaviour in selfservice settings: a systematic review. Obes Rev 14, 187-196.

18. Libotte E, Siegrist M \& Bucher T (2014) The influence of plate size on meal composition. Literature review and experiment. Appetite 82, 91-96.

19. Wansink B, Painter JE \& Lee YK (2006) The office candy dish: proximity's influence on estimated and actual consumption. Int J Obesity (Lond) 30, 871-875.

20. Bar-Hillel M \& Dayan E (2011) Nudge to nobesity II: menu positions influence food orders. Judgm Decis Mak 6, 333-342.

21. Sinclair SE, Cooper M \& Mansfield ED (2014) The influence of menu labeling on calories selected or consumed: a systematic review and meta-analysis. J Acad Nutr Diet 114, 1375-1388.

22. Campos S, Doxey J \& Hammond D (2011) Nutrition labels on pre-packaged foods: a systematic review. Public Health Nutr 14, 1496-1506.

23. Hollands GJ, Shemilt I, Marteau TM, et al. (2013) Altering micro-environments to change population health behaviour: towards an evidence base for choice architecture interventions. BMC Public Health 13, 1218.

24. Liberati A, Altman DG, Tetzlaff J, et al. (2009) The PRISMA statement for reporting systematic reviews and meta-analyses of studies that evaluate health care interventions: explanation and elaboration. PLoS Med 6, e1000100.

25. Kahn BE \& Wansink B (2004) The influence of assortment structure on perceived variety and consumption quantities. J Consum Res 30, 519-533. 
26. Academy of Nutrition and Dietetics (2012) Evidence Analysis Manual: Steps in the Academy Evidence Analysis Process. Chicago: Research and Strategic Business Development, Academy of Nutrition and Dietetics.

27. Levitz LS (1976) The susceptibility of human feeding behavior to external controls. In Obesity in Perspective, NIH publication no. $75-708$, pp. 53-60 [G Bray, editor]. Washington, DC: US Government Printing Office.

28. Musher-Eizenman DR, Young KM, Laurene K, et al. (2010) Children's sensitivity to external food cues: how distance to serving bowl influences children's consumption. Health Educ Behav 37, 186-192.

29. Engell D, Kramer M, Malafi T, et al. (1996) Effects of effort and social modeling on drinking in humans. Appetite 26, 129-138.

30. Wansink B \& Hanks AS (2013) Slim by design: serving healthy foods first in buffet lines improves overall meal selection. PLOS ONE 8, e77055.

31. Meyers AW, Stunkard AJ \& Coll M (1980) Food accessibility and food choice. A test of Schachter's externality hypothesis. Arch Gen Psychiatry 37, 1133-1135.

32. van Kleef E, Otten K \& van Trijp HC (2012) Healthy snacks at the checkout counter: a lab and field study on the impact of shelf arrangement and assortment structure on consumer choices. BMC Public Health 12, 1072.

33. Levy DE, Riis J, Sonnenberg LM, et al. (2012) Food choices of minority and low-income employees: a cafeteria intervention. Am J Prev Med 43, 240-248.

34. Thorndike AN, Sonnenberg L, Riis J, et al. (2012) A 2-phase labeling and choice architecture intervention to improve healthy food and beverage choices. Am J Public Health 102, 527-533.

35. Meiselman HL, Hedderley D, Staddon SL, et al. (1994) Effect of effort on meal selection and meal acceptability in a student cafeteria. Appetite 23, 43-55.
36. Schwarz J, Riis J, Eibel B, et al. (2012) Inviting consumers to downsize fast-food portions significantly reduces calorie consumption. Health Aff 31, 399-407.

37. Wansink B \& Hanks AS (2014) Calorie reductions and withinmeal calorie compensation in children's meal combos. Obesity (Silver Spring) 22, 630-632.

38. Maas J, de Ridder DT, de Vet E, et al. (2012) Do distant foods decrease intake? The effect of food accessibility on consumption. Psychol Health 27, Suppl. 2, 59-73.

39. Marteau TM, Ogilvie D, Roland M, et al. (2011) Judging nudging: can nudging improve population health? BMJ $\mathbf{3 4 2}$, d228.

40. Strack F \& Deutsch R (2004) Reflective and impulsive determinants of social behavior. Pers Soc Psychol Rev 8, 220-247.

41. Ducrot P, Julia C, Méjean C, et al. (2015) Impact of different front-of-pack nutrition labels on consumer purchasing intentions: a randomized controlled trial. Am J Prev Med.

42. Blumenthal-Barby JS \& Burroughs H (2012) Seeking better health care outcomes: the ethics of using the 'nudge'. $A m \mathrm{~J}$ Bioeth 12, 1-10.

43. Gold A \& Lichtenberg P (2012) Don't call me 'nudge': the ethical obligation to use effective interventions to promote public health. Am J Bioeth 12, 18-20.

44. Huang CJ \& Baum ML (2012) Nudge ethics: Just a game of billiards? Am J Bioeth 12, 22-24.

45. Potts M, Verheijde JL \& Rady MY (2012) When a nudge becomes a shove. Am J Bioeth 12, 40-42.

46. Hoefkens C, Lachat C, Kolsteren P, et al. (2011) Posting pointof-purchase nutrition information in university canteens does not influence meal choice and nutrient intake. Am J Clin Nutr 94, 562-570. 